\title{
Senior Research Scholars in China CDC's National Immunization Program
}

\author{
Miao Wang'; Yanmin Liu'; Tingting Yan ${ }^{1}$; Lance Rodewald ${ }^{1}$; Zhijie An ${ }^{1}$; Zundong Yin ${ }^{1, *}$
}

China CDC's National Immunization Program's Senior Research Scholars Program (NIP-SRSP) is a program created to foster the professional development of early-to-mid-career immunization experts, strengthen knowledge exchange and cooperation between national and provincial/prefectural-level CDCs, and deepen scientific and programmatic capacity of the National Immunization Program and provincial/prefectural immunization programs. Initiated in September 2019, the NIP-SRSP recruits immunization professionals for a 12-to-18-month experience in Beijing who have worked at the ViceSenior professional level or above and are recommended by their provincial/prefectural CDC supervisor and leadership. The first cohort of National Immunization Program Senior Research Scholars successfully completed the program and were recognized by CDC Director, Academician George F. Gao at a commencement ceremony on August 25, 2021.

\section{SELECTION PROCESS}

The program selection process is designed to ensure a good match between scholars' professional development interests and the national and provincial/prefectural immunization program needs and project opportunities. Selection starts with recommendations from prospective scholars' CDC supervisors and leadership. Successful candidates have a deep affinity for their career in immunization with fulfilling working experiences, excellent interpersonal skills, and strong teamwork spirit, and they are well organized and motivated to contribute to the mission of the National Immunization Program while in Beijing and to their home CDC upon completion of the program. Scholars must be less than 45 years of age when they start the program and have good English language skills. Their work experience should include conducting and managing studies and research projects.

\section{TRAINING METHODS}

SRSP combines theory and practice into a 12-to-18month full-time experience in Beijing that includes weekly immunization seminars; participating and teaching in conferences on infectious diseases, vaccines, epidemiology, evidence-based medicine, and pharmacovigilance; and participation in the Chinese Vaccinology Course (CNVAC), which is organized by the University of Chinese Academy of Sciences (with a curriculum that includes a global overview of vaccines, immunization strategies for prevention and control of vaccine-preventable diseases, vaccine development and preclinical research, vaccine production processes and analytics, vaccine regulation, immunization policy making, challenges of immunization and vaccinology, and communications with the public and media).

Scholars complete one or more scientific or programmatic projects that align professional background, training needs, and goals of the scholar with the needs of the National Immunization Program. Scholars will be assigned to the appropriate branch of the immunization program for project mentorship and supervision. Currently, assignments are separated into three branches: 1) the Evidence-Based DecisionMaking for Immunization Branch, which provides scientific support for the Technical Working Groups of the National Immunization Advisory Committee (NIAC) by conducting research on the methodology of evidence-based decision-making for immunization planning and providing methodological support for the formulation of technical guidelines for immunization; 2) the Vaccination and Immunization Services Branch, which conducts vaccination coverage surveillance, evaluation of vaccination service technologies, Immunization Information System development and use, programmatic policy and strategy development, technical guidelines and standards development, and school entry vaccination record check guidelines and evaluation; and 3) the Adverse Event Following Immunization (AEFI) Surveillance Branch, which 
conducts pharmacovigilance on all aspects of vaccine safety through a rigorous safety monitoring program jointly managed by China CDC and the National Medical Products Administration, identification and analysis of potential vaccine safety signals, formulation of vaccine safety technical guidelines, and monitoring vaccine-related public opinion.

$\mathrm{Du}$ Wen from Guizhou Provincial CDC and Wu Jing from Jiangxi Provincial CDC are the first graduates of this new program. Du Wen matched with the AEFI Branch to work with Dr. Li Keli, the branch chief. Ms. Du worked on the following: 1) monitoring the safety of China's new coronavirus disease 2019 (COVID-19) vaccines while they were under the Emergency Use Authorization in preparation for routine use; 2) evaluation of the National Regulatory Authority for vaccines to strengthen the capacity of AEFI surveillance and ensure that China meets or exceeds the World Health Organization's quality criteria; 3) updating the national AEFI surveillance guidelines with the most recent scientific evidence on vaccine safety monitoring; and 4) upgrading AEFI surveillance modules in Immunization Information Systems. Wu Jing matched with the Vaccination and Immunization Services Branch to work with Branch Chief Yu Wenzhou and deputy Cao Lei. Ms. Wu worked on the following: 1) a national level vaccination catch-up campaign to ensure that children who missed vaccinations due to the COVID-19 pandemic could be fully vaccinated - a campaign that immunized $94.4 \%$ of children who had missed the nearly 90 million doses of vaccines in total in the mainland of China (1); 2) the national COVID-19 vaccination campaign that protected frontline workers, working-age adults, older adults, and adolescents from COVID-19, providing over 1.9 billion doses of vaccine by the time that Ms. Wu graduated from the scholars program; and 3) an evaluation of COVID-19 vaccination breakthrough cases to characterize their severity and implications for effective use of COVID19 vaccines. Upon completing the program, the two scholars returned to their home programs to bring their experiences back to their home CDCs.

\section{EXAMINATION AND EVALUATION}

Senior Research Scholars are evaluated on successful completion of their project or projects by the National Immunization Program and provincial/prefectural CDC leadership. Scholars are expected to prepare at least one manuscript for publication as first author in a core domestic or international journal level, to have participated in senior-level meetings, and to have presented at one or more national professional meetings. Upon successful completion of the Program, scholars will be awarded the Certificate of Accomplishment in the Senior Research Scholar Project of the National Immunization Program (Figure 1) and will receive an evaluation report on their work and experience. Scholars are to provide feedback to SRSP to help improve this relatively young program so that it meets the training and experience needs of early-to-mid-career professionals and helps advance their careers and the field of vaccines and immunization.

\section{LOOKING FORWARD}

The SRSP will become a mainstream program supported by the Chinese Centers for Disease Control and Prevention's National Immunization Program. SRSP supports professional development of early-tomid-career immunization experts from provincial/prefectural level CDCs but is also designed to bring innovation and new ideas to China CDC. China's vaccine circulation law, which was enacted December 2019, requires provincial-level administrative divisions (PLADs) to implement a core immunization program, but it also allows PLADs to expand their program beyond the required core. For example, the Shanghai immunization program has discontinued the use of the live, oral poliovirus vaccine and has changed to an all inactivated poliovirus vaccination schedule in anticipation to similar changes nationally in future years; several PLADs are implementing an insurance-based vaccine injury compensation program to integrate compensation for program and non-program vaccine injuries into a
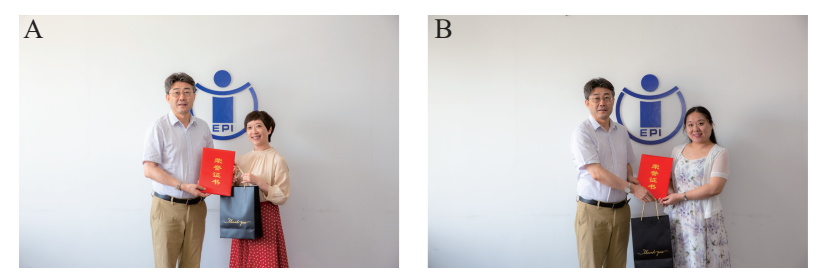

FIGURE 1. On August 25, 2021, China CDC Director George F. Gao awarded Certificates of Accomplishment to the first cohort of China CDC's National Immunization Program's Senior Research Scholar Program fellows (A) Du Wen and (B) Wu Jing. 
single, evidence-based program; and several PLADs and prefecture-level cities have integrated influenza vaccine for school children into their program. Scholars will be able to bring their experience with such innovations to the national level, enriching the entire program.

The SRSP is poised to support new scholars every year into one-to-two-year programs of theory and practice. For future cohorts, all of the branches of the National Immunization Program will be available for scholars to consider. SRSP will ultimately cover all PLADs and sub-provincial cities in our country, strengthening and integrating scientific and programmatic immunization leadership to ensure success of China's vital mission to protect children, adolescents, and adults from vaccine-preventable diseases.

doi: $10.46234 / \mathrm{ccdcw} 2021.223$

\# Corresponding author: Yin Zundong, yinzd@chinacdc.cn.

${ }^{1}$ National Immunization Program, Chinese Center for Disease Control and Prevention, Beijing, China.

Submitted: September 27, 2021; Accepted: October 07, 2021

\section{REFERENCES}

1. Wu J, Yu WZ, Cao L, Cao LS, Rodewald L, Ye JK, et al. Effectiveness of catch-up vaccinations after COVID-19 containment-China, 2020 China CDC Wkly 2020;2(50):968 - 74. http://dx.doi.org/10.46234/ ccdcw2020.262. 